\title{
THE DIAGNOSIS OF CEREBRAL PALSY IN INFANCY
}

\author{
BY \\ KAREL BOBATH and BERTA BOBATH \\ From the Cerebral Palsy Centre, London
}

(RECEIVED FOR PUBLICATION APRIL 20, 1956)

Many workers in the field of cerebral palsy are agreed that an early diagnosis is of great importance and that better and quicker results can be expected from early treatment. Very early treatment, if possible before the age of 6 months, gives a child the best chance of improvement for the following reasons:

(1) In the infant, motor behaviour is largely reflex, and the higher centres of the central nervous system are only beginning to mature. During the first six to 12 months of the baby's life the use of the neural mechanism and its patterns can be influenced and directed more easily than later on. Gooddy and McKissock (1951) have stressed the importance of age for adjustment and learning in cases of brain lesions, and say:

'Whatever the underlying anatomical basis for residual function may be, we note that the facility with which the patient can readjust himself to cerebral damage seems to be related to his age on the one hand and to the rate of progress, if any, of the causal lesion on the other. The younger brain, like bodily tissues and organs, seems more adaptable and "plastic" than the older.'

(2) An infant with cerebral palsy does not usually show appreciable degrees of spasticity, and movements are not yet interfered with by the resistance of opposing hypertonic muscles. Movements can therefore be encouraged more easily at this stage, and early treatment, by facilitating normal movements, often prevents the development of spasticity (Bobath and Bobath, 1954, 1955).

(3) In untreated cases of cerebral palsy spasticity develops and increases in strength, its typical distribution resulting in the well known abnormal postures of the patient. If the patient maintains these abnormal postures for long, contractures and deformities are likely to occur. These are never seen in the infant and young child and can be prevented by early treatment.

(4) Cerebral palsy is a sensori-motor disorder. Movements are the response to sensory stimulation. They give the child a great variety of sensory experiences. To return to Gooddy and McKissock (1951):

'There is not a standard brain with all its functions set and localized for use. The brain stands ready for training. It learns, is trained, by appreciating input, sensation. It can then effect voluntarily what it has before done involuntarily.'

A child's intellectual development is dependent on both environmental stimulation and the appreciation of his own body as a result of proprioceptive stimulation. During the first year of a child's life his mental growth is dependent on an unimpeded development of his motor abilities. A child deprived of the ability to raise his head, to look around, to reach out for an object and bring it to his mouth. to acquire hand-eye coordination, and to sit up and move around to explore his environment, cannot but fail to advance in his mental development. If, in the severely handicapped child, the inability to move is not overcome at an early age, mental retardation will result and may in time become indistinguishable from mental deficiency.

\section{Intelligence in Children with Cerebral Palsy}

The correct or even approximate assessment of mental endowment of babies with cerebral palsy is extremely difficult and no satisfactory solution to this problem has so far been found. Collis (1953, 1954) has attempted to find tests for the detection of mental deficiency in infants with cerebral palsy as early as at 4 months of age. According to Collis, the unmodified reflex behaviour of an infant at that age has to be regarded as symptomatic of mental deficiency. To quote Collis (1954):

'Where the four month old child shows little or no diminution of the reflex quality of his activity and (apart from growth) little change in his appearance, mental deficiency can be presumed.'

All tests described by Mrs. Collis are tests of motor ability only, and are quite unsuitable for the detection of mental defect. The tests serve only to 
separate the moderate from the severe cases of cerebral palsy, rejecting the latter from treatment on the grounds of mental deficiency. If accepted, they will exclude from early treatment just those severely affected children who are in most urgent need of it. As long as we have no reliable means of assessing intelligence at an early age it seems better to give every young child a chance of treatment. In the course of treatment it will often be seen whether a child can make proper use of his improving motor abilities and in this way we may come to a better understanding and more correct assessment of the child's potential mental ability. Even if the child should later on prove to be mentally defective, treatment may help him to become, physically at least, partly independent, and deformities may be prevented.

\section{Definition of the Condition}

As ideas about the nature of cerebral palsy differ widely, we shall first define our concept of the condition and discuss some points which form the basis of the tests upon which we rely for the early detection of cerebral palsy.

Cerebral palsy is not one condition but a group of conditions, the result of abnormal brain development or brain damage. We find in all cases an impaired motor function and an abnormal degree and distribution of muscle tone. This may be associated with speech defects (dysarthria or aphasia), hearing defects (of the conductive or perceptive type), visual defects (subnormal vision, field defects or ocular motor defects) and mental defect.

It is beyond the scope of this paper to go into the problems of incidence, aetiology and classification of cerebral palsy. These subjects have been discussed in great detail by Asher and Schonell (1950), Holoran (1952), Balf and Ingram (1955) and others. It is difficult enough to devise a satisfactory classification of cerebral palsy in older children. In the very young ones, under 12 months of age, in whom definite signs of cerebral palsy such as spasticity or involuntary athetoid movements are not yet seen, a correct classification is rarely possible. For instance, in a quadriplegic child one side of the body is usually more affected than the other. When very young, the child may only show definite abnormal signs on the more affected side, so that we may arrive at a diagnosis of hemiplegia. Some babies may show spasticity fairly early but may develop athetosis at about 18 months of age, while others may seem limp and ataxic and only develop pronounced spasticity later on. Other infants may show spasticity in the legs and no abnormal signs in the trunk and arms until later on when they try to sit up and use their hands; the initial diagnosis of spastic paraplegia may then have to be changed into one of diplegia.

The difficulty of classification at an early age is enhanced by the fact that many cases of cerebral palsy are of a mixed character. Whereas pure cases of spasticity are frequent among the hemiplegics and diplegics, quadriplegics often show athetosis, and most athetoids show various admixtures of spasticity or ataxia. In spite of these difficulties we should be able to make a diagnosis of cerebral palsy at an early age, though a differential diagnosis may be impossible.

We give below some figures which may be a pointer towards the relative significance of some aetiological factors. They are of 190 consecutive cases on our files of which we could obtain reliable birth histories by personal interview. Of these 190 cases, 33 showed normal birth histories and five had acquired cerebral palsy in early childhood. Of the remaining 152 cases, 36 were born prematurely. They, and the remaining 116 cases, showed the following birth histories:

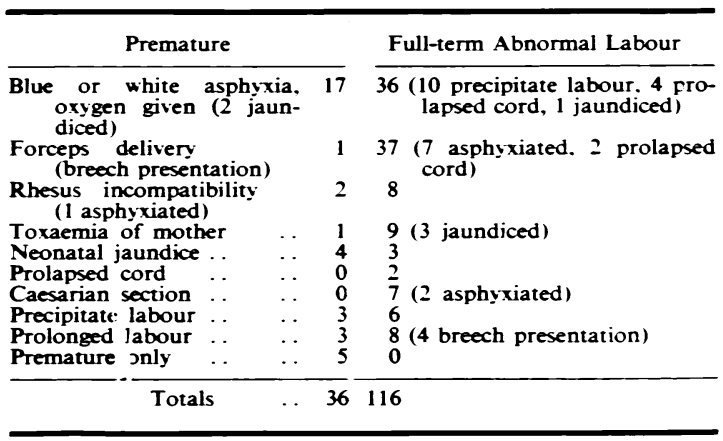

It will be seen from the above figures that the factors at birth most responsible for cerebral palsy in this group of 152 cases were asphyxia, forceps delivery, prematurity and prolonged and precipitate labour.

The number and the percentage of cases in the present series attributable to these four conditions as well as the figures given by Asher and Schonell are shown in the accompanying table:

\begin{tabular}{|c|c|c|c|}
\hline $\begin{array}{l}\text { Causal } \\
\text { Factor }\end{array}$ & $\begin{array}{l}\text { No. of } \\
\text { Cases }\end{array}$ & $\begin{array}{r}\text { Present Series } \\
\text { (152 Cases) }\end{array}$ & $\begin{array}{l}\text { Asher and } \\
\text { Schonell }\end{array}$ \\
\hline $\begin{array}{lll}\text { Asphyxia } & \cdots & \text {. } \\
\text { Forceps } & \cdots & \text { Prematurity }\end{array}$ & $\begin{array}{l}62 \\
38 \\
36\end{array}$ & $\begin{array}{l}40 \% \\
25 \%\end{array}$ & \multirow{2}{*}{$\begin{array}{c}38 \% \\
24 \% \\
\text { No compar } \\
\text { able figures } \\
15 \%\end{array}$} \\
\hline $\begin{array}{l}\text { Prolonged and pre } \\
\text { cipitate labour }\end{array}$ & 30 & $20 \%$ & \\
\hline
\end{tabular}




\section{Some Relevant Points of Early Normal Motor Development}

In the normal child motor development takes place in an orderly sequence (Gesell and Amatruda, 1947; McGraw, 1943; Thomas and Saint-Anne Dargassies, 1952). The normal baby shows a preponderantly flexor tone during the first two months of life. The limbs are held in flexion and resist passive extension. The distribution of flexor tone is strictly symmetrical. Even when kicking the baby does not extend his legs fully until he is about 6 months old. Under normal circumstances extensor tone develops gradually from the second month onwards, when the baby raises his head in prone lying and extends his spine against gravity, supporting himself first on his forearms and later on his extended arms. At about 6 months of age the baby lifts his buttocks off the support when lying on his back, supporting himself on his shoulders and feet, thus extending his spine and hips. In this way he prepares for the upright posture, for sitting, standing and walking. The influence of certain primitive reflexes, the tonic or static reflexes of Magnus (1926), can be seen in the normal baby. For instance, the asymmetrical tonic neck reflex shows itself in typical attitudes of the baby, face turned to one side and the 'face-arm' extended, the opposite arm flexed. One can observe this attitude more clearly and more frequently from the fifth week onwards, that is, at a time when extensor tone develops. The influence of the tonic reflexes diminishes after 4 months and then gradually disappears. Tonic reflexes, however, are never strong in the normal baby. They do not produce a stiffening of the baby's limbs and they do not interfere with his movements.

\section{The Early Motor Development of Children with Cerebral Palsy}

Like the normal baby, infants with cerebral palsy show at first a preponderance of flexor tone. It is only at the stage of developing extensor tone that the child with cerebral palsy becomes spastic. The speed and development of spasticity and its degree depend on the severity of the case. With the onset of spasticity we can observe an increase in the strength of the aforementioned tonic reflexes. Instead of disappearing, they gain in strength, and in time they may dominate the child's motor activities. They are coexistent with spasticity and prevent the further maturation of the child's motor behaviour. They are responsible for the typical distribution of spasticity throughout the body musculature. They produce changes of tone in predictable patterns when the position of the whole body in space is changed, or when the relation of the head to the body is changed.

In trying to make an early diagnosis, we search for signs of activity of tonic reflexes before they attain their full strength. Early diagnosis is easy in the severe cases in which spasticity and tonic reflexes develop quickly and strongly. In the milder cases they appear later, are more insidious and have to be provoked by special handling. In the athetoid patient they are less constant, and show themselves in the form of intermittent tonic spasms of variable strength. They have been observed by Ingram (1955) who calls them 'dystonic attacks'.

The tonic reflexes, which have been described in some detail in previous papers (Bobath and Bobath, $1954,1955)$, include the tonic neck reflexes, the tonic labyrinthine reflexes and the positive supporting reaction. Only these, which are most important for diagnostic purposes, will be mentioned in this paper.

Asymmetrical Tonic Neck Reflex. This consists of an increase of extensor tone in the limbs to which the face is turned, and an increase of flexor tone in the limbs to which the skull is turned. The 'face-arm and leg' may extend, the 'skull-arm and leg' flex.

Tonic Labyrinthine Reflexes. These produce maximal flexor tone throughout the whole body in lying prone (face downwards), and maximal extensor tone in the supine position (face looking upwards).

Positive Supporting Reaction. This produces rigid extension of the leg, making it a 'pillar for weight-bearing' when the ball of the foot touches the ground.

As mentioned before, the increase of the strength of these reflexes goes together with the development of extensor spasticity. In some, usually in the spastic children, we can observe a slow and gradual increase of tone over a prolonged period of time. Only in the most severe cases is spasticity already present and strong at a very early age. These children, when lying on the back, show stiffly extended legs which resist passive flexion and abduction. They show retraction of head and neck and strong asymmetrical tonic neck reflexes. The hands are usually fisted, the arm flexed at the elbows and abducted at the shoulders. If the arms are passively moved forward at the shoulder, extended at the elbow, strong resistance will be encountered. However, the majority of infants do not show 
definite signs of spasticity at a very early age. This is especially so with paraplegic children, or with diplegic children whose upper extremities are little affected. These children may appear quite normal until they try to sit up or crawl. A few children with very mild affection may show spasticity only when they attempt to stand or walk.

Athetoid children do not usually show involuntary movements before the twelfth or eighteenth month. of age and sometimes not before they are 2 years old These babies give the impression of being hypotonic. They may hardly move at all when they are very young, lying quietly on the back. When picked up they have no control over head and trunk; they are just floppy. The legs are usually flexed and widely abducted with an exaggerated dorsiflexion of the ankles. Some athetoid children wave their arms about aimlessly and seem to be unable to direct them in reaching out for an object. This initial state of limpness or flaccidity begins to alternate with transient phases of stiffening which becomes stronger and more frequent as the child develops, and when he tries to move and maintain his posture against gravity.

In order to arrive at an early diagnosis the task before the doctor is to differentiate between a general retardation of the child's motor development and early signs of spasticity or intermittent spasms. For this purpose we need special tests which provoke signs of spasticity while they are still latent. In other words, during examination the child is handled in a way which provokes abnormal spastic reactions.

\section{Tests for Examination}

A number of tests will be described, none of which alone is more than a pointer to a

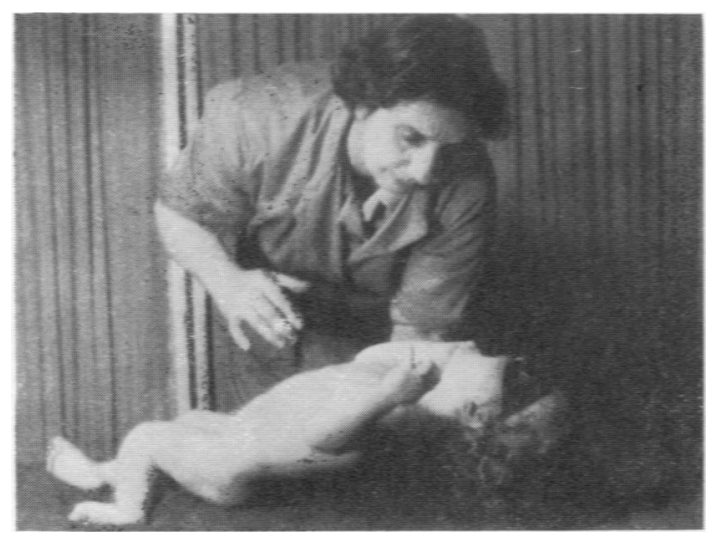

Fig. 1.-Extensor spasticity in the extensor position. diagnosis. The certainty of the diagnosis is in direct proportion to the number of tests found positive.

Testing in the Supine Position. This is the position of maximal extensor spasticity due to tonic labyrinthine reflex activity.

Testing for Retraction of Head and Neck. The examiner's hand is placed behind the child's head and an attempt is made to lift the child into the sitting position. One can feel the head and neck pressing backwards or, in the more severe cases, the whole spine may arch backwards. The arms, instead of moving forward on the chest as in the normal child, are retracted at the shoulders (Fig. 1). Head and neck retraction explain the child's inability to lift the head actively in the supine position, a preliminary to sitting up.

Testing for Retraction of the Shoulders. The examiner grasps the arms of the child at the elbows and moves them forward and across the child's chest. Resistance to this movement can be felt and the arms pull backwards. This explains the child's inability to bring his arms forward and to pull himself up to the sitting position, especially as he is unable to lift his head.

TESTING FOR THE INFLUENCE OF THE ASYMMETRICAL Tonic Reflex on the Arms. As already described, the infant usually lies on his back with the arms in abduction and flexed at the elbows. The examiner turns the head of the child to one side and holds it in this position for a few seconds. The arm to which the face is turned may then extend spontaneously. while the contralateral arm becomes more flexed (Fig. 2). In some cases the 'face-arm' may not extend spontaneously, but passive extension of the elbow will be less resisted than before the face was turned

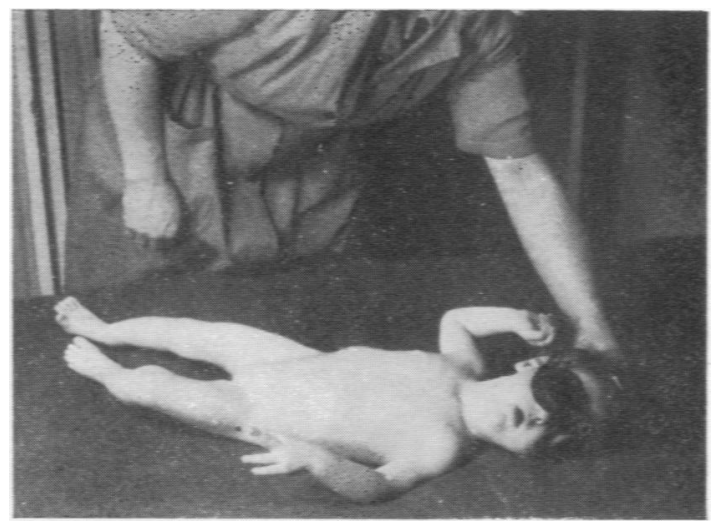

Fig. 2.-Asymmetrical tonic neck reflex. 
towards that side, while passive extension of the 'skull-arm' will be more resisted. If asymmetrical tonic neck reflexes are present, it is difficult, or impossible, for the child to grasp an object with the hand to which his face is turned, while he can do so with the hand to which the skull is turned. This means that he cannot look at the object when grasping it. Asymmetrical tonic neck reflexes explain the child's inability to approximate both hands in the midline, his inability to bring his hands to his mouth and of acquiring hand-eye coordination.

Testing for Absence of Neck Righting Reflex. On turning the head of an infant to one side, the body should follow the head and the child turn over to that side. This is a normal reaction up to 3 years of age. In severe cases of cerebral palsy the child will remain on his back and only an asymmetrical tonic neck reflex will occur. Such children are unable to turn over by themselves. In less severe cases the body may turn but the uppermost arm remains retracted. Such children may be able to turn over to their side, but only slowly and with difficulty.

Testing for Extensor Spastictity of Legs. The examiner grasps the legs of the child below the knees and moves them quickly upward to flex them against the abdomen. Resistance to this movement is noted. If the legs are suddenly released, they extend immediately and may adduct and even cross. If one leg only is flexed passively, the other leg may extend stiffly and rotate inward with plantiflexed foot (crossed extension reflex). In some children, however, both legs may flex when one is passively flexed. Resistance to passive flexion of the legs and the child's inability to keep them flexed after they are released, denotes extensor spasticity at the hips,

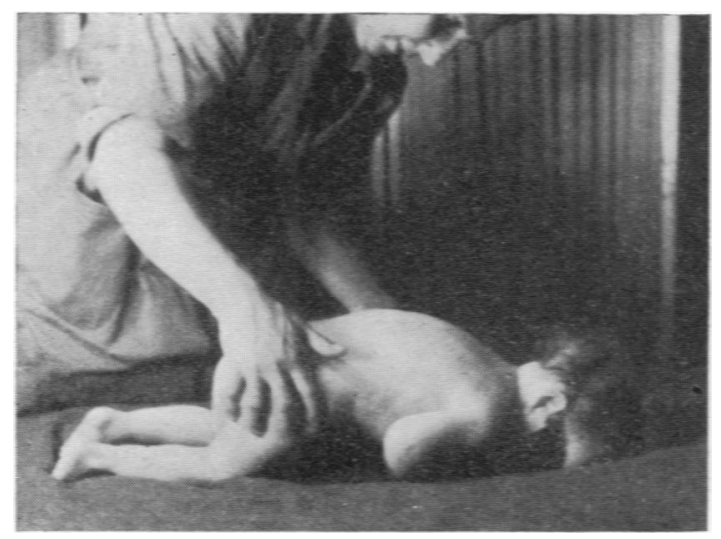

Fig. 3.-Absence of automatic turning of the head to the side in the prone position. and this explains the child's difficulties in sitting up and his tendency to fall backwards when sitting. A normal child rarely falls backward when he is yet unable to sit unsupported. It also explains the child's inability to kick in the normal way.

Testing for AdDuctor SPasticity OF the Legs. Adductor spasticity is usually combined with extensor spasticity. It is, therefore, best tested with the legs in extension. The examiner grasps the legs below the knees and moves them quickly apart. Resistance to the movement is noted.

Tests in the Prone Position. This is the position of maximal flexor spasticity due to tonic labyrinthine reflex activity.

Testing for Absence of Protective SideTURNING OF HEAD. A normal baby when lifted up and placed on his abdomen will turn the head to one side. This is a protective reflex, already present at birth. If flexor spasticity is marked a child with cerebral palsy will not do this but will remain face downwards on the support (Fig. 3). For this reason children with involvement of trunk and upper extremities cannot tolerate the prone position as they cannot keep the air passages free. We consider the inability to tolerate this position a valuable sign at a time when other symptoms may not yet be present.

Testing for Flexor Spasticity of ARms. The child with cerebral palsy when lying on the abdomen shows flexion and adduction of the arms, the hands placed under the chest. The examiner grasps the hands of the child and moves the arms upwards so that they lie extended at the side of the head. Note is taken of the resistance to this movement. On releasing the arms they will return to their initial flexed and adducted position.

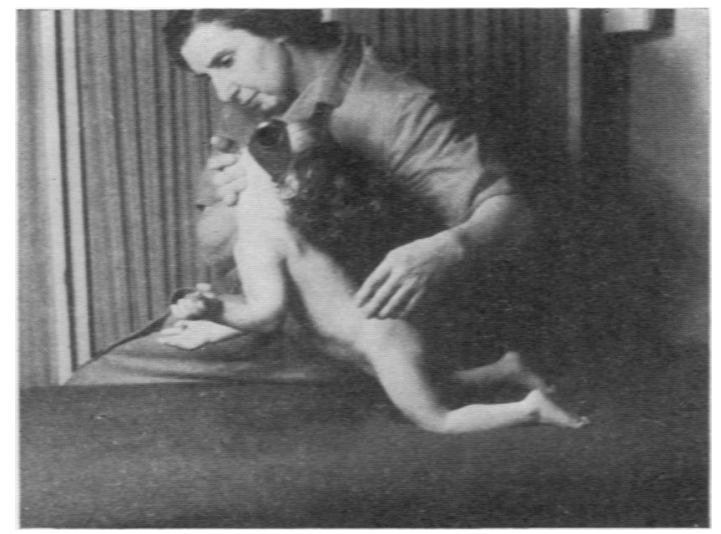

Fig. 4.-Absence of extension of the arms to support the weight of the body. 
Another test is that of raising the child's head passively with the examiner's hand under the child's chin. In some cases resistance to this movement will be felt, that is, pressure downwards of the head against the examiner's hand. The normal reaction of a child of 4 months and onwards to raising the head is a protective extension of the arms, the hands being placed on the support. The child with cerebral palsy will draw up the arms in flexion and be unable to support his body weight on his hands. The hips may flex simultaneously (Fig. 4).

Testing for Extensor SPasticity of Legs. As mentioned before, flexor spasticity is maximal in the prone position, and in severe cases extension of the hips may be impossible so that the child cannot be placed on his abdomen. However, if the hips can be extended, this extension produces extensor spasticity at the knees and feet, though the trunk, neck and arms remain flexed. The examiner lifts the child's hips off the support, holding the pelvis around the iliac crests. If extensor spasticity is marked, the legs extend stiffly and it may be difficult to flex them passively. In less severe cases, the legs may flex spontaneously but only slowly and after considerable delay (Fig. 5).

One can also test for extensor spasticity by flexing the knees while holding the pelvis down to the support. This must be done with the thighs adducted, extensor spasticity being stronger with adducted legs. Resistance to passive flexion of the knees is then encountered. If the hips are released the knees may show less resistance to passive flexion, but the hips will flex as well (Fig. 6).

Flexor spasticity of the trunk and arms and extensor spasticity of the legs prevent the child from raising the head, and from getting on to his hands and knees to crawl.

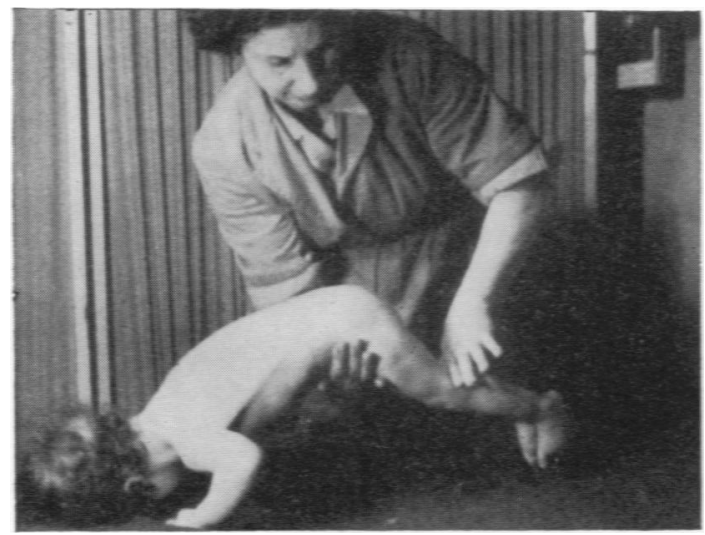

Fig. 5.-Resistance of the legs to passive flexion.
Tests in the Upright Posture. The last tests are in the upright posture.

Testing for Extensor Spastictity OF the Legs. The examiner holds the child under the axillae in the air and lowers him slowly towards the support. The legs may then extend and the feet point downwards. In some cases the legs may adduct and even cross. On touching the support the child may support his body-weight and stand on his toes, with the legs rigidly extended. Some children with moderate spasticity may show reflex stepping, but cross the legs when doing so (Fig. 7).

Other children, usually those who seem flaccid and lie supine with abducted legs, may draw up the legs in flexion when held up in the air. They will not support their weight on their legs when their feet touch the support. The examiner then 'bounces' the child several times in quick succession on his feet. An increase of extensor tone in the legs may then be noted, which may become stronger with each 'bounce' and result in a temporary stiffening of the legs. These children usually develop extensor spasticity in the legs later on.

The 'Landau' Reflex. The examiner holds the child free in the air supported under the abdomen. A normal child, from the age of 6 to 8 months onwards, will lift the head and extend his spine and legs (this reaction has disappeared by the age of 3 years). If the examiner flexes the head passively the whole body will flex. The 'Landau' reflex is usually absent in the child with cerebral palsy. When held in the air, the child will neither raise his head nor extend his spine and hips. The knees, however, may be extended if extensor spasticity is marked. The absence of the 'Landau' reflex is due to the inhibiting influence of flexor spasticity on the

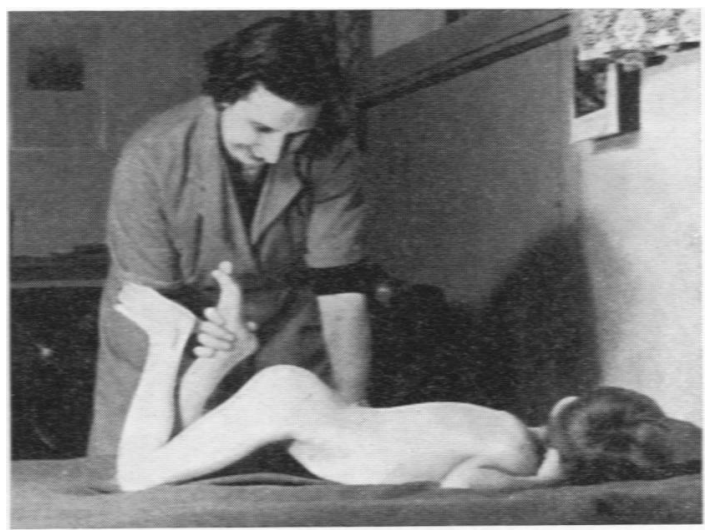

Fig. 6.-Flexion of the knees produces flexion of the hips. 


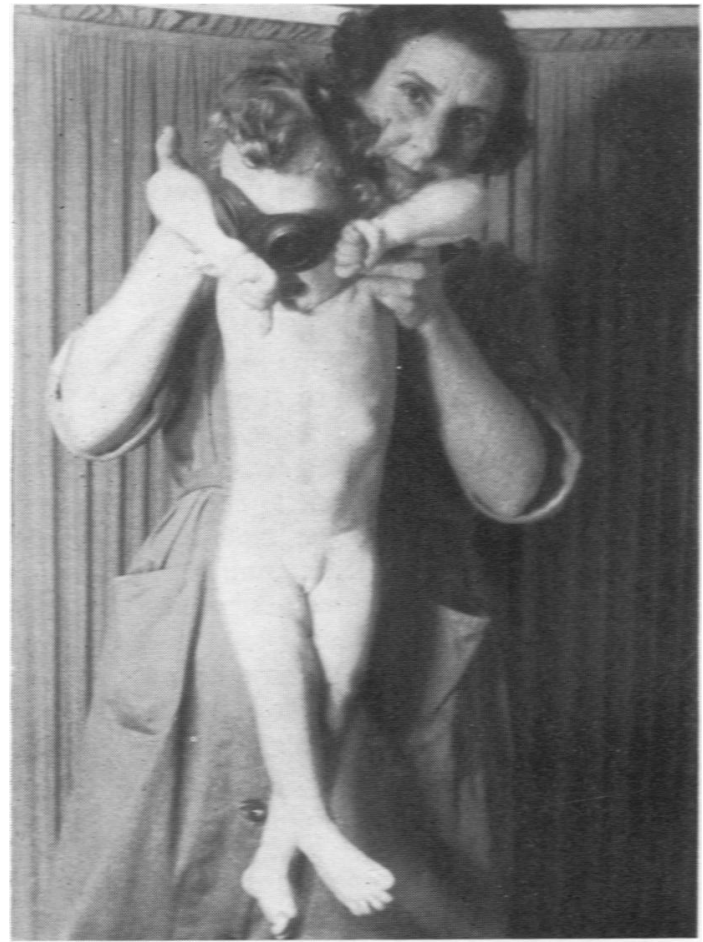

FKG. 7. - When the child is held free in the air, the feet point downwards and the legs sometimes cross.

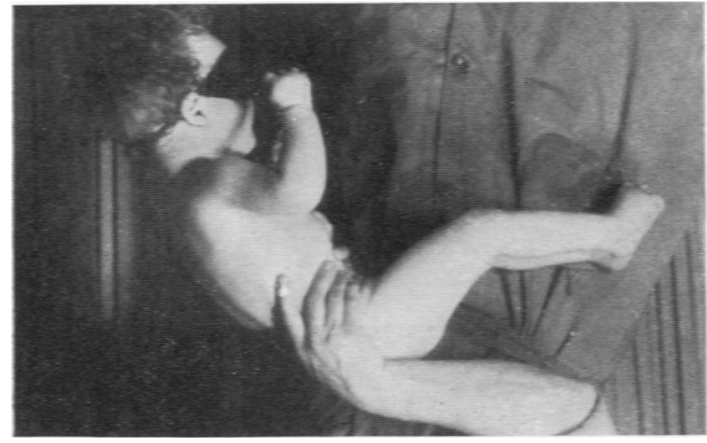

FIG. 8.-Absence of protective extension of the arms. head and trunk when the head is facing downwards (tonic labyrinthine reflex).

Protective Extension of the Arms. This reaction, as well as the one above, has been described by Schaltenbrand (1927). The latter was called 'Sprungbereitschaft'. As in testing for the 'Landau' reflex, the examiner lifts the child up, holding him around the waist, and then lowers him slowly downwards towards the support. A normal child from 4 to 6 months of age onwards will extend his arms and hands to reach out for the support. A child with cerebral palsy will draw up his arms in flexion (Fig. 8). In less severe cases the arms may extend protectively when the head touches the support.

\section{Summary}

A number of tests have been described to assist in the early recognition of cerebral palsy. They are based on the work of Magnus (1926) and Schaltenbrand (1927), and demonstrate the presence of abnormal postural reflex activity. The diagnosis is based on the interpretation of the child's motor disabilities in terms of abnormal postural reflex activity.

We wish to thank Dr. E. Ellis, Medical Director of the Percy Hedley School for Spastic Children, for his advice and valuable criticism in the preparation of this paper.

\section{REFERENCES}

Asher, P. and Schonell. F. E. (1950). Archives of Disease in Childhood, 25. 360.

Balf C. L and Ingram. T T S (1955) Brit med J 2 163

Bobath, B. (1954). Physiotherapy, 40, 259, 295, 326, 368.

(1955). Ibid., 41. 146.

Bobath, K. and Bobath, B. O. (1954). Brit. orthoptic J., 11. 88.

C. (1955). Cerebral Palsy Rev.. 16. No. 5. p. 4

Collis. E. (1953). Lancet, 1, 416

Gesell. A. and Amatruda, C. S. (1947). Developmental Diagnosis, 2nd ed. New York

Gooddy, W. and McKissock, W. (1951). Lancet, 1, 481.

Holoran, I. M. (1952). Brit. med. J.. 1. 214.

Ingram. T. T. S. (1955). Archives of Disease in Childhood, 30, 85.

Magnus, R. (1926). Lancet, 2, 531, 585.

McGraw, M. B. (1943). The Neuromuscular Maturation of the Human Infant. New York.

Thomas, A. and Saint-Anne Dargassies (1952). Études neurologiques sur le nouveau-ne et le jeune nourrisson. Paris. 